\title{
Versatility of the translational machinery during stress: changing partners to keep dancing
}

\author{
Fátima Gebauer ${ }^{1}$ \\ ${ }^{1}$ Gene Regulation, Stem Cells and Cancer Programme, Centre for Genomic Regulation (CRG-UPF), Dr Aiguader 88, 08003-Bar- \\ celona, Spain \\ Cell Research (2012) 22:1634-1636. doi:10.1038/cr.2012.102; published online 3 July 2012
}

Cap-dependent translation is initiated by the binding of eIF4E to the cap structure at the $5^{\prime}$ end of mRNAs. During hypoxic stress, global translation decreases because eIF4E is inactivated. In a recent article in Nature, Lee and colleagues show that residual hypoxic translation is maintained by a specialized isoform of eIF4E, which binds to target mRNAs in complex with a hypoxia-induced RNP.

Translation of most messenger RNAs in the cell is initiated by binding of eukaryotic initiation factor (eIF) $4 \mathrm{E}$ to the $\mathrm{m}^{7} \mathrm{GpppN}$ cap structure at the mRNA 5 ' end. Multiple eIF4E isoforms have been described in a variety of organisms along the evolutionary scale, but their functions remain for the most part obscure [1]. Translation is drastically reduced upon low oxygen tension (hypoxia) by mechanisms that inhibit the function of eIF4E. Cell function under hypoxia, however, is maintained by a significant degree of "residual" translation. In a recent article in Nature, Lee and colleagues show that the eIF4E homologue 4E2, in complex with the transcription factor HIF- $2 \alpha$ and the RNA-binding protein RBM4, sustains cap-dependent translation during hy-

Correspondence: Fátima Gebauer Tel: +34-93-3160120; Fax: +34-93-3969983

E-mail: fatima.gebauer@crg.eu poxia [2]. Thus, by using a specialized isoform of eIF4E in complex with a hypoxia-induced RNP, the translational machinery preserves selective protein synthesis during stress (Figure 1).

The hypoxia inducible factor (HIF) family of transcription factors maintain cellular oxygen homeostasis. HIF- $2 \alpha$ is induced under hypoxia and activates the expression of epidermal growth factor receptor (EGFR), a factor that confers growth advantage to hypoxic tumor cells. Lee and colleagues first show that the accumulation of EGFR is independent of the transcriptional activity of HIF-2 $\alpha$. First, EGFR protein accumulates in cells treated with transcription inhibitors; second, no changes in the steady state amounts of EGFR mRNA are detected upon hypoxia; and third, depletion of HIF-1 $\beta$, a factor required for HIF transcriptional activity, has no effect on EGFR expression. These results pointed to a surprising direct role of HIF-2 $\alpha$ in EGFR mRNA translation, a notion reinforced by the finding that HIF- $2 \alpha$ associates with polysomes.

The authors further show that HIF$2 \alpha$ associates to a region of EGFR 3' UTR that is necessary and sufficient to drive translation of reporters in a HIF$2 \alpha$-dependent manner. HIF- $2 \alpha$ does not contain discernible RNA-binding motifs, raising the possibility that RNA binding is mediated by interactions with bridge RNA-binding proteins. An unbiased search for HIF- $2 \alpha$ interactors identified RBM4, a protein involved in translational control $[3,4]$. RBM4 interacts with the HIF- $2 \alpha$-responsive region of EGFR 3' UTR, and its depletion results in dissociation of HIF- $2 \alpha$ from EGFR mRNA, indicating that RBM4 recruits HIF-2 $\alpha$ to the 3' UTR of the EGFR transcript. Furthermore, depletion of RBM4 abrogates the hypoxic translation of endogenous EGFR or EGFR reporters without affecting the levels of HIF- $2 \alpha$. Thus, RBM4 mediates the effects of HIF-2 $\alpha$ in hypoxic translation of EGFR mRNA.

But the HIF-2 $\alpha /$ RBM4 complex regulates more than just EGFR translation. Silencing of HIF-2 $\alpha$ or RBM4 considerably reduced the rate of global hypoxic translation as measured by ${ }^{35} \mathrm{~S}-$ methionine incorporation into nascent proteins. UV crosslinking of RNAs associated with the HIF-2 $\alpha /$ RBM 4 complex identified CGG or CG boxes in hundreds of transcripts, a hallmark of RBM4 binding [5]. Consistent with the relevance of the RBM4 site, mutation of a specific CGG box in the $3^{\prime}$ UTR of EGRF abrogated hypoxia-induced translation of a reporter. The CGG, however, was not sufficient to confer hypoxic translation, as it must be embedded in a region that the authors refer to as the rHRE (RNA Hypoxia Response Element).

How does the HIF-2 $\alpha /$ RBM4 com- 


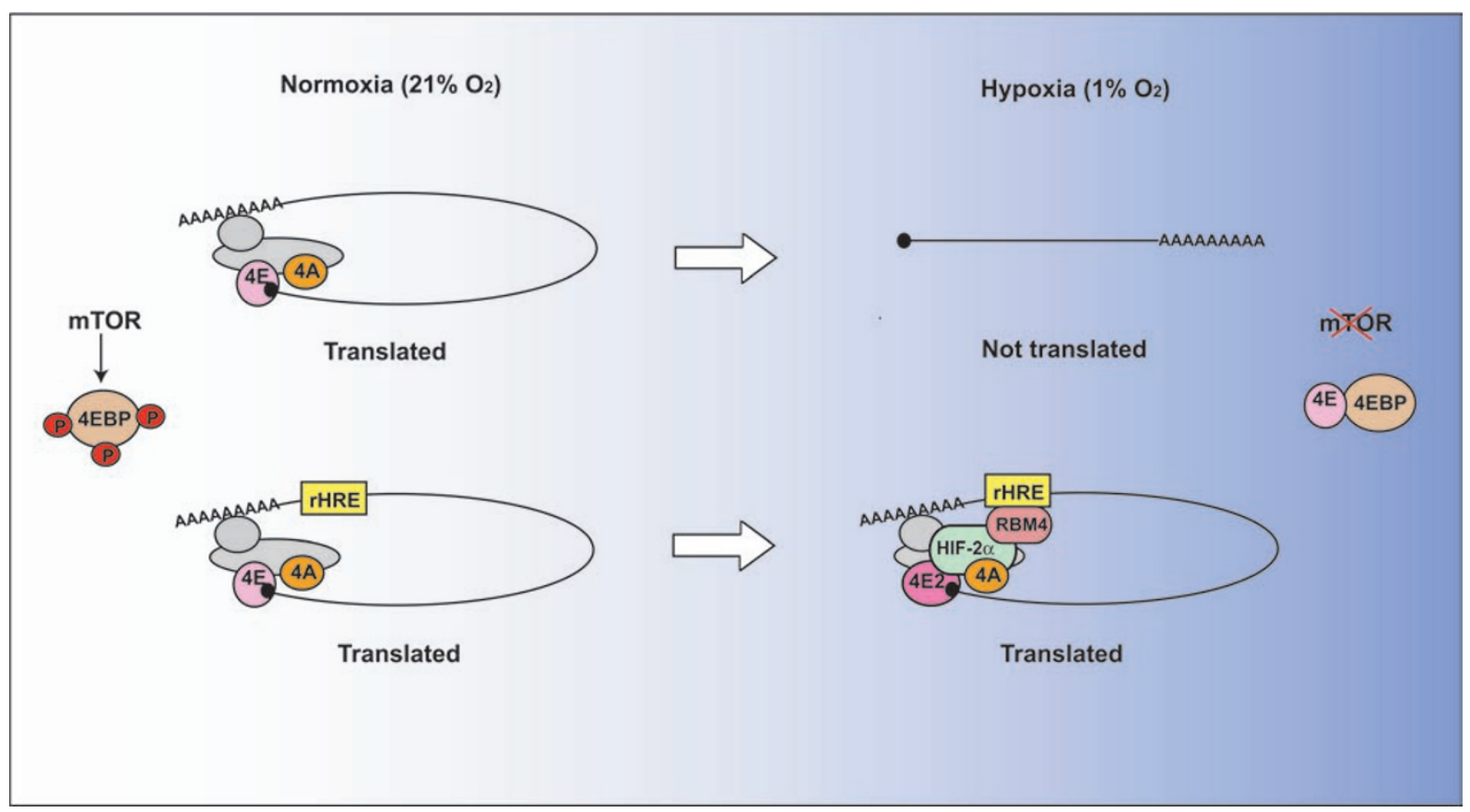

Figure 1 Translational control under hypoxia. In normal growing conditions (normoxia, $21 \% \mathrm{O}_{2}$ ), translation of the vast majority of transcripts is initiated via a mechanism where elF4E binds to the cap structure (black circle) together with elF4A and additional factors (elF4G and PABP, gray ovals) to promote ribosome recruitment. In this condition, 4EBP remains hyperphosphorylated and inactivated by mTOR. Under hypoxia $\left(1 \% \mathrm{O}_{2}\right)$, inactivation of mTOR leads to accumulation of hypophosphorylated 4EBP, which binds and inactivates elF4E leading to reduced global translation. Hypoxia also induces the expression of HIF-2 $\alpha$, which interacts with RBM4, elF4A and elF4E2. Binding of RBM4 to the RNA Hypoxia Response Element (rHRE) allows the recruitment of this complex to a subset of transcripts and their translation by an elF4E2-dependent mechanism.

plex stimulate global translation under hypoxia? The cap-binding complex, composed of eIF4E, the RNA helicase eIF4A and the scaffolding protein eIF4G, is a common target for translation regulation. Testing interactions with components of this complex revealed that neither HIF-2 $\alpha$ nor RBM4 interact with eIF4E or eIF4G, but they do interact with eIF4A and the eIF4E isoform $4 \mathrm{E} 2$, suggesting that the HIF-2 $\alpha /$ RBM4 complex binds to an alternative capbinding assembly. eIF4E2, also known as 4E-homologous protein (4EHP) [6], does not interact with eIF4G and binds only weakly to 4EBP1, a factor that binds and inhibits eIF4E under hypoxia [2, 7-9]. The low affinity of eIF4E2 for 4EBP1 explains its resistance to inactivation in this metabolic condition. HIF- $2 \alpha$ and RBM4 are retained in $\mathrm{m}^{7} \mathrm{GTP}$ cap columns under hypoxic, but not normoxic, conditions and silencing of eIF4E2 prevents this binding. Thus, eIF4E2 tethers the HIF-2 $\alpha /$ RBM complex to the cap structure.

Importantly, silencing of eIF4E2, but not eIF4E, prevented the translation of rHRE-containing targets and dramatically reduced the amount of ${ }^{35} \mathrm{~S}$ methionine incorporation into nascent proteins under hypoxia. Conversely, silencing of eIF4E, but not eIF4E2, inhibited basal translation of HIF- $2 \alpha$ targets and reduced global translation under normoxia. These results uncovered a switch from eIF4E- to eIF4E2dependent translation in response to oxygen tension (Figure 1). They further identified eIF4E2 as an activator of translation.

One question that emerges from this study is why eIF4E2 does not function in general translation during normoxia. The explanation may lie on the observation that eIF4E2 has a weak binding affinity for the cap structure and, in normal conditions, it cannot compete with eIF4E for cap binding [10]. During hypoxia, however, eIF4E is inactivated by $4 \mathrm{EBP}$, increasing the chances that eIF4E2 binds the cap. Increased local concentration of eIF4E2 on specific targets promoted by interactions with RNA-binding proteins [2], or posttranslational modifications of eIF4E2 [11] may contribute to translational stimulation under particular conditions. Finally, interaction of eIF4E2 with specific initiation factor isoforms could also provide versatility in translational control.

The stimulatory role of eIF4E2 in translation during hypoxia contrasts with the known function of this factor in other biological contexts. During Drosophila embryo axis formation, for instance, d4EHP is recruited to caudal and nanos mRNAs by the RNA-binding 
proteins Bicoid and Brat, respectively, and inhibits the translation of these transcripts $[12,13]$. The capacity of d4EHP to inhibit translation has been attributed to its inability to bind eIF4G, which has a primarily scaffolding function during translation initiation and supports multiple interactions for ribosome recruitment. In agreement with this, human eIF4E2 does not interact with eIF4G during hypoxia. How can then eIF4E2 stimulate translation? One possibility is that a specific eIF4G isoform acts together with eIF4E2 during hypoxia. Indeed, precedent for functional eIF4G isoforms exist in the literature. The eIF4G homologue protein DAP5/p97 is known to support translation of select mRNAs during endoplasmic reticulum stress and has also been proposed to function in non-stressed cells $[14,15]$. Although DAP5 lacks the eIF4E-binding domain, it could retain the capacity to interact with eIF4E homologues. Alternatively, novel proteins could substitute for the scaffolding function of eIF4G during translation initiation. One candidate for this is HIF- $2 \alpha$ itself, which strongly interacts with eIF4A. Delineation of the composition of the cap-binding complex associated with eIF4E2 during hypoxia may clarify these issues. Nevertheless, the results of Lee and colleagues illustrate the plasticity of the translational machinery to adapt to changing metabolic conditions.

\section{Acknowledgments}

I thank Juan Valcárcel for critical comments on this manuscript. Work in my lab is supported by BFU2009-08243 and Consolider CSD2009-00080 from MICINN.

\section{References}

1 Hernández G, Vazquez-Pianzola P. Functional diversity of the eukaryotic translation initiation factors belonging to eIF4 families. Mech Dev 2005; 122:865-876.

2 Uniacke J, Holterman CE, Lachance $\mathrm{G}$, et al. An oxygen-regulated switch in the protein synthesis machinery. $\mathrm{Na}$ ture 2012; 486:126-129.

3 Lin JC, Hsu M, Tarn WY. Cell stress modulates the function of splicing regulatory protein RBM4 in translation control. Proc Natl Acad Sci USA 2007; 104:2235-2240.

4 Lin JC, Tarn WY. RNA-binding motif protein 4 translocates to cytoplasmic granules and suppresses translation via argonaute 2 during muscle cell differentiation. J Biol Chem 2009; 284:3465834665.

5 Ray D, Kazan H, Chan ET, et al. Rapid and systematic analysis of the RNA recognition specificities of RNA-binding proteins. Nat Biotechnol 2009; 27:667670.

6 Rom E, Kim HC, Gingras AC, et al. Cloning and characterization of 4EHP, a novel mammalian eIF4E-related capbinding protein. J Biol Chem 1998; 273:13104-13109.

7 Tee AR, Tee JA, Blenis J. Characterizing the interaction of the mammalian eIF4E-related protein 4EHP with 4E-
BP1. FEBS Lett 2004; 564:58-62.

8 Joshi B, Cameron A, Jagus R. Characterization of mammalian eIF4Efamily members. Eur J Biochem 2004; 271:2189-2203.

9 Hernández G, Altmann M, Sierra JM, et al. Functional analysis of seven genes encoding eight translation initiation factor 4E (eIF4E) isoforms in Drosophila. Mech Dev 2005; 122:529-543.

10 Zuberek J, Kubacka D, Jablonowska A, et al. Weak binding affinity of human 4EHP for mRNA cap analogs. RNA 2007; 13:691-697.

11 Okumura F, Zou W, Zhang DE. ISG15 modification of the eIF4E cognate 4EHP enhances cap structure-binding activity of 4EHP. Genes Dev 2007; 21:255-260.

12 Cho PF, Poulin F, Cho-Park YA, et al. A new paradigm for translational control: inhibition via 5'-3' mRNA tethering by Bicoid and the eIF4E cognate 4EHP. Cell 2005; 121:411-423.

13 Cho PF, Gamberi C, Cho-Park YA, Cho-Park IB, Lasko P, Sonenberg N. Cap-dependent translational inhibition establishes two opposing morphogen gradients in Drosophila embryos. Curr Biol 2006; 16:2035-2041.

14 Nousch M, Reed V, Bryson-Richardson RJ, Currie PD, Preiss T. The eIF4Ghomolog p97 can activate translation independent of caspase cleavage. $R N A$ 2007; 13:374-384.

15 Lewis SM, Cerquozzi S, Graber TE, Ungureanu NH, Andrews M, Holcik M. The eIF4G homolog DAP5/p97 supports the translation of select mRNAs during endoplasmic reticulum stress. Nucleic Acids Res 2008; 36:168-178. 